\title{
Impact of COVID-19 Restrictions on Demographics and Outcomes of Patients Undergoing Medically Necessary Non- Emergent Surgeries During the Pandemic
}

\author{
Adrienne B. Shannon ${ }^{1}$ (1) Jeffrey L. Roberson ${ }^{1} \cdot$ Luke Keele $^{1} \cdot$ Tina Bharani $^{1} \cdot$ Yun Song $^{1} \cdot$ \\ John T. Miura ${ }^{2}$ Rachel R. Kelz ${ }^{2} \cdot$ Daniel T. Dempsey ${ }^{3} \cdot$ Lee A. Fleisher $^{4} \cdot$ Ronald P. DeMatteo $^{2}$ • \\ Giorgos C. Karakousis ${ }^{2}$
}

Accepted: 5 January 2021 / Published online: 28 January 2021

(C) Société Internationale de Chirurgie 2021

\begin{abstract}
Background The COVID-19 pandemic has resulted in large-scale healthcare restrictions to control viral spread, reducing operating room censuses to include only medically necessary surgeries. The impact of restrictions on which patients undergo surgical procedures and their perioperative outcomes is less understood.

Methods Adult patients who underwent medically necessary surgical procedures at our institution during a restricted operative period due to the COVID-19 pandemic (March 23-April 24, 2020) were compared to patients undergoing procedures during a similar time period in the pre-COVID-19 era (March 25-April 26, 2019). Cardinal matching and differences in means were utilized to analyze perioperative outcomes.

Results 857 patients had surgery in 2019 (pre-COVID-19) and 212 patients had surgery in 2020 (COVID-19). The COVID-19 era cohort had a higher proportion of patients who were male $(61.3 \%$ vs. $44.5 \%, P<0.0001)$, were White $(83.5 \%$ vs. $68.7 \%, P<0.001)$, had private insurance $(62.7 \%$ vs. $54.3 \%, p 0.05)$, were ASA classification 4 $(10.9 \%$ vs. $3 \%, P<0.0001)$, and underwent oncologic procedures $(69.3 \%$ vs. $42.7 \%, P<0.0001)$. Following $1: 1$ cardinal matching, COVID-19 era patients $(N=157)$ had a decreased likelihood of discharge to a nursing facility (risk difference-8.3, $P<0.0001$ ) and shorter median length of stay (risk difference-0.6, $p$ 0.04) compared to preCOVID-19 era patients. There was no difference between the two patient cohorts in overall morbidity and 30-day readmission.

Conclusions COVID-19 restrictions on surgical operations were associated with a change in the racial and insurance demographics in patients undergoing medically necessary surgical procedures but were not associated with worse postoperative morbidity. Further study is necessary to better identify the causes for patient demographic differences.
\end{abstract}

Supplementary Information The online version contains supplementary material available at (https://doi.org/10.1007/s00268021-05958-z)

Adrienne B. Shannon

adrienne.shannon@pennmedicine.upenn.edu

1 Department of Surgery, Hospital of the University of Pennsylvania, 3400 Spruce Street, 5 Silverstein, Philadelphia, PA 19104, USA

2 Division of Endocrine and Oncologic Surgery, Department of Surgery, Hospital of the University of Pennsylvania, Philadelphia, PA, USA
3 Division of Gastrointestinal Surgery, Department of Surgery, Hospital of the University of Pennsylvania, Philadelphia, PA, USA

4 Department of Anesthesiology and Critical Care, Perelman School of Medicine, University of Pennsylvania, Philadelphia, PA, USA 


\section{Introduction}

Severe acute respiratory syndrome (SARS)-CoV-2 or COVID-19 has rapidly expanded worldwide due to its high virulence resulting in international public lockdowns [1-7]. Following the first case in the United States, many hospitals instituted practices restricting elective surgical procedures to help prevent the spread of the contagion to patients and healthcare workers and to preserve medical resources [8-16]. On March 18, 2020, the Centers for Medicare and Medicaid Services (CMS) announced that elective procedures should be delayed, which prompted 33 states to issue independent guidance and mandates for the utilization of hospital resources [10]. At the Hospital of the University of Pennsylvania, in an effort to both prevent spread of infection and to preserve hospital resources, a policy was implemented in mid-March limiting surgical procedures to those deemed medically necessary by the surgeon, including operations for severe symptomatology and for cancer treatment [17-19].

These restrictions resulted in a significant reduction in our operative census and various hospital personnel, including operating room staff and perioperative nurses, were redeployed during this time period. We sought to investigate if these changes would impact perioperative outcomes and the patient demographics of those undergoing medically necessary procedures. While attention has been given to the disparate proportion of COVID-19 cases and resulting deaths among Black and minority patients, particularly in areas of higher poverty and lower education rates, there is presently limited information on the potential impact of the pandemic on the non-COVID-19 health problems [20-24]. We hypothesized that minority and disadvantaged socioeconomic populations may have been disproportionately impacted by COVID-19 pandemic restrictions with respect to undergoing medically necessary surgical procedures.

\section{Study design}

\section{Study population selection}

Adult ( $\geq 18$ years) patients who underwent elective surgical procedures performed at the Hospital of the University of Pennsylvania between March 23, 2020 and April 24, 2020 were identified for inclusion into the COVID-19 era cohort. The study time period was determined based on the implementation of the CMS restrictions and continued until prior to relaxation of restrictions during the approximate resurgence time instituted by our institution. "Elective" procedures were defined as those that were scheduled as non-emergent, medically necessary and for which patients arrived at the hospital the morning of surgery prior to their procedure start time. Patients admitted from the emergency department prior to surgery were excluded. Routine calculation of the medically necessary, time-sensitive (MeNTS) scoring system was not implemented until May 4, 2020; prior to this time, the decision for medically necessary procedures was made by the surgeon with oversight by respective departments and divisions [18].

Urology, obstetrics and gynecology, or breast oncology procedures were excluded to reduce the likelihood of gender bias that these specialties could introduce on other factors of this study, namely socioeconomic factors, as prior studies have shown that men are more likely than women to be uninsured [25]. Minor procedures (i.e., endoscopic) were excluded from the study. Similarly, patients who met these criteria who underwent elective surgical procedures from March 25, 2019 to April 26, 2019 were identified as the pre-COVID-19 era cohort. Two patients undergoing palliative procedures were excluded from the COVID-19 era cohort who expired within 30 days of surgery. The study was approved by the Institutional Review Board of the University of Pennsylvania (protocol \#842,962) and in accordance with the Health Insurance Portability and Accountability Act.

\section{Matching strategy}

In our study, we exactly matched 2019 and 2020 patients on ICD-10 procedure code categories based on the 73 ICD10 procedure codes that occurred in 2020. Patients with ICD-10 procedure code categorization in 2019 that did not align with those identified from 2020 were not included in matching. An optimal matching algorithm based on integer programming to pair patients who were most similar in terms of measured covariates was used. We matched using cardinality matching [26] with fine balance-a method of constraining two groups to be balanced on a particular variable without restricting matching on the variable within individual pairs - to nearly exact balance nominal variables [27]. We used cardinality matching to find the largest sample of matched patient pairs that have covariate differences in means smaller than a pre-specified difference, and the marginal distributions of surgical specialty are near finely balanced (Supplementary Table 1, Supplementary Table 2).

\section{Statistical analysis}

Overall morbidity was computed using a composite of the following events: ventilation requirements $>48 \mathrm{~h}$, new hemodialysis needs, total parental nutrition requirements, blood transfusion, need for reoperation, need for 
percutaneous drainage of abscess, wound infection, pneumonia, acute kidney injury, sepsis or septic shock, myocardial infarction, stroke, and thromboembolic events. To assess the quality of the matches, we computed the standardized difference for each covariate [28-30]. Differences in study outcomes before and after matching in each cohort (2020 and 2019) were compared using paired tests. McNenmar's test [30, 31] was used for binary outcomes, and m-estimates [32, 33] were used for continuous outcomes after matching. All hypothesis tests were twosided with a significance threshold of $P<0.05$. Statistical analyses were conducted using STATA/IC 13.1 statistical software and $\mathrm{R}$ statistical software 3.3.2 [34, 35]. All matching analyses were conducted using the designmatch package 18 in $\mathrm{R}$.

\section{Results}

\section{Patient and clinical characteristics of pre-COVID-19 and COVID-19 surgical patients}

From March 25, 2019 to April 26, 2019, there were 857 $(80.2 \%)$ patients who underwent elective surgical procedures, and from March 23, 2020 to April 24, 2020, there were $213(19.8 \%)$ patients who underwent medically necessary surgical procedures (Table 1). Overall, $71.7 \%$ of patients identified as White. When compared to patients from the pre-COVID-19 era cohort, the COVID-19 era cohort had higher proportions of patients 50-80 years of age, whereas the pre-COVID-19 era cohort had a higher proportion of patients who were $<50$ years of age and $>$ 80 years of age (Table 1). The COVID-19 era cohort had a higher proportion of patients who were male and with private insurance as compared to pre-COVID-19 era cohort patients. Of the COVID-19 era cohort patients, a higher proportion of patients was White race (83.5\% vs. $68.7 \%)$ and a lower proportion of patients was Black non-Hispanic (9\% vs. $19 \%$ ), Asian (2.4\% vs. $3.3 \%$ ), or Hispanic $(2.4 \%$ vs. $4.9 \%$ ) when compared to pre-COVID-19 era cohort patients $(P 0.001)$.

When examining clinical characteristics of patients, COVID-19 era cohort patients had more comorbidities and were more likely to have ASA classification 3 or 4 as compared to pre-COVID-19 era patients (Table 2). The majority of patients in both cohorts had a pre-operative 30-day history and physical visit with their surgical team, but $5.2 \%$ of COVID-19 era cohort patients had this visit done via telemedicine. A higher proportion of COVID-19 era cohort patients was admitted post-operatively to the hospital compared to pre-COVID-19 era cohort patients. Lastly, the frequency of procedures related to an oncologic diagnosis was significantly greater in the COVID-19 era cohort when compared to pre-COVID-19 era cohort.

\section{Perioperative outcomes}

Of the ICD-10 procedure code categories identified from 2020 patients (Supplementary Table 2), 391 (45.6\%) of all pre-COVID-19 era cohort patients were identified to have corresponding code categories to the 212 COVID-19 era cohort patients with available code categories and were utilized to analyze perioperative outcomes. When comparing these selected COVID-19 era cohort patients to preCOVID-19 era cohort patients with ICD-10 code categorization amenable to matching, patients had a higher likelihood of overall morbidity (risk difference-12.6, $P<0.0001)$ and discharge to their home with home services (risk difference-8.2, $P$ 0.03) but had a lower likelihood of being discharged to a skilled nursing facility (risk difference-4.1, $P$ 0.01) (Table 3). COVID-19 era cohort patients were also more likely to have a longer postoperative median LOS (risk difference-0.8, $P$ 0.04).

Following 1:1 cardinal matching, COVID-19 era patients $(N=157)$ no longer demonstrated an increased risk of overall morbidity (risk difference-8.9, $P 0.10$ ) or discharge to home with home services (risk difference-3.2, $P 0.42$ ) but continued to have a decreased likelihood of being discharged to a skilled nursing facility (risk difference-8.3, $P<0.0001)$. Additionally, COVID-19 era patients had shorter median LOS (risk difference-0.6, p 0.04) as compared to pre-COVID-19 era patients. Similar to unadjusted analysis, 30-day readmission rates were no different between the two cohorts following matching (risk difference-7.6, $P$ 0.07).

\section{Discussion}

As a result of the COVID-19 pandemic, hospitals nationwide have canceled all non-essential surgeries during surges of COVID-19 infections; at the Hospital of the University of Pennsylvania, this restriction on non-essential procedures occurred during the months of March and April [17-19]. During this time period, only procedures which were considered by the surgeon to be medically necessary were conducted. Our investigation aimed to understand how this change impacted the typical elective procedure patient census and to study the postoperative outcomes related to surgical procedures during the pandemic.

The implementation of pandemic restrictions and adoption of medically necessary justification for surgery resulted in a substantial decrease $(75 \%)$ in the elective procedure patient census. Of the patients who underwent 
Table 1 Patient characteristics of patients undergoing elective surgical procedures at a single insitution during a five-week period in 2019 (preCOVID-19) and during a five-week period in 2020 in which restrictions were in place for the COVID-19 pandemic

\begin{tabular}{|c|c|c|c|}
\hline Patient characteristics & $2019($ Pre-COVID-19) $\quad N=857(80.2 \%)$ & $2020($ COVID-19) $N=213(19.8 \%)$ & $P$ value \\
\hline Age & & & $0.05^{*}$ \\
\hline $18-29$ years & $66(7.7)$ & $11(4.7)$ & \\
\hline $30-39$ years & $94(11.0)$ & $17(8.0)$ & \\
\hline $40-49$ years & $106(12.4)$ & $23(10.9)$ & \\
\hline $50-59$ years & $173(20.2)$ & $48(22.6)$ & \\
\hline $60-69$ years & $210(24.5)$ & $65(30.7)$ & \\
\hline $70-79$ years & $153(17.9)$ & $44(20.8)$ & \\
\hline$\geq 80$ years & $55(6.4)$ & $5(2.4)$ & \\
\hline Sex & & & $<0.0001^{*}$ \\
\hline Male & $381(44.5)$ & $131(61.3)$ & \\
\hline Female & $476(55.5)$ & $82(38.7)$ & \\
\hline Race/ethnicity & & & $0.001 *$ \\
\hline White & $589(68.7)$ & $178(83.5)$ & \\
\hline Black & $163(19.0)$ & $19(9.0)$ & \\
\hline Asian & $28(3.3)$ & $5(2.4)$ & \\
\hline Hispanic & $42(4.9)$ & $5(2.4)$ & \\
\hline Other/unknown & $35(4.1)$ & $6(2.8)$ & \\
\hline Marital status & & & 0.20 \\
\hline Single & $225(26.3)$ & $42(19.3)$ & \\
\hline Married/domestic partner & $534(62.3)$ & $146(68.9)$ & \\
\hline Divorced/separated & $46(5.4)$ & $15(7.1)$ & \\
\hline Widowed & $51(6.0)$ & $10(4.7)$ & \\
\hline Other/unknown & $1(0.1)$ & $0(0.0)$ & \\
\hline Insurance & & & $0.05 *$ \\
\hline None & $8(0.9)$ & $0(0.0)$ & \\
\hline Private & $465(54.3)$ & $134(62.7)$ & \\
\hline Medicare & $320(37.3)$ & $71(33.5)$ & \\
\hline Medicaid & $58(6.8)$ & $6(2.8)$ & \\
\hline Government & $6(0.7)$ & $2(0.9)$ & \\
\hline
\end{tabular}

*Indicates significance

elective procedures during the pandemic, we noted that patients were primarily $50-80$ years old as opposed to $<$ 50 and $>80$ years of age; this possibly could be secondary to an increased prevalence of cancers in this age population relative to younger patients. Among octogenarian patients, the perceived risk of COVID-19 infection may have dissuaded patients and providers from pursuing surgery. COVID-19 era patients had increased comorbidities with higher rates of cardiac problems and immune compromise. While these patients perhaps had the most medical necessity in the cohort due to symptomatology, they were also at the highest risk for complications and associated mortality from a COVID-19 infection [3, 4, 20, 36-38]. Though none of our surgical patients contracted COVID-19 in the perioperative period, these cases pose significant challenges in decision-making for providers and patients due to the complex risk/benefit balance.

Notably, a substantial racial difference was observed between COVID-19 era patients and pre-COVID-19 era patients. Minority patients were significantly less likely to undergo surgical procedures during the COVID-19 pandemic. Black patients have been shown to have proportionally higher rates of COVID-19 infection as compared to White patients with three-fold higher infection rates in predominantly Black counties as compared to predominantly White counties [20-24]. Our study suggests that Black and other minority patients may not just be at increased risk of COVID-19 and its associated morbidity but may also be at risk of having other aspects of their healthcare compromised during the pandemic. 
Table 2 Clinical characteristics of patients undergoing elective surgical procedures at a single insitution during a five-week period in 2019 (preCOVID-19) and during a five-week period in 2020 in which restrictions were in place for the COVID-19 pandemic

\begin{tabular}{|c|c|c|c|c|c|c|c|}
\hline Clinical characteristics & 2019 & 2020 & $P$ value & & 2019 & 2020 & $P$ value \\
\hline ASA classification & & & $<0.0001^{*}$ & Pre-operative HP visit & & & $<0.0001 *$ \\
\hline 1 & $41(4.8)$ & $0(0.0)$ & & None & $14(1.6)$ & $1(0.5)$ & \\
\hline 2 & $403(47.0)$ & $73(34.4)$ & & Clinic & $843(98.4)$ & $200(93.9)$ & \\
\hline 3 & $387(45.2)$ & $116(54.7)$ & & Telemedicine & $0(0.0)$ & $11(5.2)$ & \\
\hline 4 & $26(3.0)$ & $24(10.9)$ & & Telephone & $0(0.0)$ & $1(0.5)$ & \\
\hline Comorbidities & & & & Primary surgical specialty & & & $<0.0001 *$ \\
\hline Prior stroke & $42(4.9)$ & $10(4.7)$ & 0.91 & Gastrointestinal & $134(15.6)$ & $29(13.7)$ & \\
\hline Coronary artery disease & $82(9.6)$ & $40(18.9)$ & $<0.0001^{*}$ & Colorectal & $54(6.3)$ & $29(13.7)$ & \\
\hline Hypertension & $426(49.7)$ & $114(53.3)$ & 0.35 & Oncologic & $184(21.5)$ & $39(18.4)$ & \\
\hline Valve disorder & $95(11.1)$ & $22(9.9)$ & 0.62 & Thoracic & $49(5.7)$ & $35(16.5)$ & \\
\hline Arrhythmia & $81(9.5)$ & $32(15.1)$ & $0.02 *$ & Vascular & $32(3.7)$ & $7(3.3)$ & \\
\hline Congestive heart failure & $52(6.1)$ & $19(8.5)$ & 0.20 & Plastic surgery & $73(8.5)$ & $10(4.7)$ & \\
\hline Prior myocardial infarction & $31(3.6)$ & $17(8.0)$ & $0.01 *$ & Otorlaryngology & $130(15.2)$ & $24(11.3)$ & \\
\hline Prior cardiac stent & $24(2.8)$ & $22(10.4)$ & $<0.0001 *$ & Cardiac & $63(7.4)$ & $24(10.9)$ & \\
\hline Peripheral arterial disease & $37(4.3)$ & $19(9.0)$ & $0.01 *$ & Neurosurgery & $100(11.7)$ & $8(3.8)$ & \\
\hline COPD & $40(4.7)$ & $9(4.3)$ & 0.79 & Orthopedics or foot & $18(2.1)$ & $8(3.8)$ & \\
\hline Cirrhosis & $4(0.5)$ & $2(0.9)$ & 0.41 & Oral maxillofacial surgery & $20(2.3)$ & $0(0.0)$ & \\
\hline Ascites & $5(0.6)$ & $2(0.9)$ & 0.56 & Secondary specailty & & & 0.10 \\
\hline Diabetes mellitus & & & $0.02 *$ & Gastrointestinal & $9(1.1)$ & $3(1.4)$ & \\
\hline IDDM & $33(3.9)$ & $16(7.6)$ & & Colorecatal & $2(0.2)$ & $0(0.0)$ & \\
\hline NIDDM & $123(14.4)$ & $20(9.4)$ & & Oncologic & $2(0.2)$ & $1(0.5)$ & \\
\hline Chronic kidney disease & $65(7.6)$ & $17(8.0)$ & 0.83 & Thoracic & $0(0.0)$ & $2(0.9)$ & \\
\hline Dialysis requirements & $15(1.8)$ & $3(1.4)$ & 0.73 & Vascular & $4(0.5)$ & $2(0.9)$ & \\
\hline Prior DVT or PE & $40(4.7)$ & $28(13.2)$ & $<0.0001^{*}$ & Plastic surgery & $7(0.8)$ & $2(0.9)$ & \\
\hline History of smoking & $260(30.3)$ & $105(49.5)$ & $<0.0001 *$ & Otolaryngology & $12(1.4)$ & $0(0.0)$ & \\
\hline Chronic steroid use & $24(2.8)$ & $13(6.1)$ & $0.02 *$ & Neurosurgery & $1(0.1)$ & $1(0.5)$ & \\
\hline Obesity (BMI $\geq 30 \mathrm{~kg} / \mathrm{m}^{2}$ ) & $345(40.3)$ & $94(43.9)$ & 0.34 & Anesthesia type & & & 0.19 \\
\hline Hemoglobin (median, IQR) & $13.6(2.1)$ & $13.7(2.1)$ & 0.54 & General & 805 (93.9) & $207(97.2)$ & \\
\hline Thrombocytopenia & $38(4.4)$ & $17(8.0)$ & $<0.0001 *$ & Monitored anesthesia care & $38(4.4)$ & $5(2.4)$ & \\
\hline Unknown & $310(36.2)$ & $35(16.5)$ & & Regional anesthesia & $3(0.4)$ & $1(0.5)$ & \\
\hline Hypoalbuminemia & $11(1.3)$ & $9(4.3)$ & $<0.0001 *$ & Local anesthesia & $11(1.3)$ & $0(0.0)$ & \\
\hline Unknown & $491(57.3)$ & $74(34.4)$ & & Surgical approach & & & $0.002 *$ \\
\hline Pre-operative WBC count & & & $<0.0001 *$ & Open procedure & $264(30.8)$ & $135(63.2)$ & \\
\hline Normal & $478(55.8)$ & $157(73.6)$ & & Minimally invasive & $87(10.2)$ & $78(36.8)$ & \\
\hline Leukopenia & $40(4.7)$ & $13(6.1)$ & & Not applicable & $506(59.0)$ & $0(0.0)$ & \\
\hline Leukocytosis & $29(3.4)$ & $8(3.8)$ & & Oncologic procedure & $366(42.7)$ & $147(69.3)$ & $<0.0001 *$ \\
\hline \multirow[t]{2}{*}{ Unknown } & $310(36.2)$ & $35(16.5)$ & & Admission to hospital & $473(55.2)$ & $166(77.8)$ & $<0.0001 *$ \\
\hline & & & & Intensive care unit admission & $103(12.0)$ & $36(16.5)$ & 0.08 \\
\hline
\end{tabular}

ASA American Society of Anesthesiologists, COPD chronic obstructive pulmonary disease, IDDM insulin-dependent diabetes mellitus, NIDDM non-insulin-dependent diabetes mellitus, $D V T$ deep venous thrombosis, $P E$ pulmonary embolus, $B M I$ body mass index, $I Q R$ interquartile range, $W B C$ white blood cell, $H P$ history and physical

* indicates significance

Additionally, we noted a difference in insurance type with a higher proportion of patients undergoing medically necessary surgeries during the pandemic having private insurance, as compared to no insurance, Medicare, and Medicaid together with the noted racial differences, these findings suggest unexpected disparities in the wake of this health crisis.

Previous studies investigating disparities related to COVID-19 have suggested that worse healthcare outcomes can result from the differences in socioeconomic factors 
Table 3 Unadjusted and adjusted patient outcome estimates of morbidity, length of stay, discharge locaiton, and 30-day readmission of patients undergoing elective surgical procedures in a five-week time period in 2019 and 2020, respectively, following 1:1 cardinal matching

\begin{tabular}{|c|c|c|c|c|c|c|c|c|}
\hline \multirow[t]{2}{*}{ Patient outcomes } & \multicolumn{4}{|c|}{ Unadjusted patient outcome estimates } & \multicolumn{4}{|c|}{ Adjusted patient outcome estimates } \\
\hline & $\begin{array}{l}2019 \\
(N=391 \\
64.8 \%)\end{array}$ & $\begin{array}{l}2020 \\
(N=212, \\
35.2 \%)\end{array}$ & $\begin{array}{l}\text { Risk } \\
\text { difference }\end{array}$ & $P$ value & $\begin{array}{l}2019 \\
(N=157 \\
50 \%)\end{array}$ & $\begin{array}{l}2020 \\
(N=157 \\
50 \%)\end{array}$ & $\begin{array}{l}\text { Risk } \\
\text { difference }\end{array}$ & $P$ value \\
\hline Morbidity & $67(17.1)$ & $63(29.7)$ & $\begin{array}{c}12.6[5.0 \\
20.1]\end{array}$ & $<0.0001^{*}$ & $35(22.3)$ & $49(31.2)$ & $\begin{array}{l}8.9[-0.9, \\
18.5]\end{array}$ & 0.10 \\
\hline $\begin{array}{l}\text { Length of stay (median, } \\
\text { IQR) }\end{array}$ & $2.4(5.0)$ & $3.0(4.0)$ & $\begin{array}{c}0.8[0.1 \\
1.6]\end{array}$ & $0.04 *$ & $3.4(5.0)$ & $2.8(5.0)$ & $\begin{array}{l}-0.6 \\
\quad[-1.2 \\
0.1]\end{array}$ & $0.04 *$ \\
\hline $\begin{array}{l}\text { Discharge to home with } \\
\text { home services }\end{array}$ & $73(18.7)$ & $57(26.9)$ & $\begin{array}{c}8.2[0.7 \\
15.7]\end{array}$ & $0.03 *$ & $36(22.9)$ & $41(26.1)$ & $\begin{array}{l}3.2[-5.7, \\
12.0]\end{array}$ & 0.42 \\
\hline $\begin{array}{l}\text { Discharge to skilled } \\
\text { nursing facility or } \\
\text { rehab }\end{array}$ & $18(4.6)$ & $1(0.5)$ & $\begin{array}{l}-4.1 \\
\quad[-6.8 \\
\quad-1.5]\end{array}$ & $0.01 *$ & $14(8.9)$ & $1(0.6)$ & $\begin{array}{l}-8.3 \\
\quad[-12.9 \\
\quad-3.4]\end{array}$ & $<0.0001 *$ \\
\hline 30-day readmission & $42(10.7)$ & $18(8.5)$ & $\begin{array}{c}-2.3 \\
{[-7.5,} \\
3.0]\end{array}$ & 0.46 & $28(17.8)$ & $16(10.2)$ & $\begin{aligned}- & 7.6 \\
& {[-15.2} \\
& 0.1]\end{aligned}$ & 0.07 \\
\hline
\end{tabular}

$I Q R$, interquartile range

*Indicates significance

and access to healthcare [39]. Though there has been a significant increase in telemedicine capabilities during this pandemic, it is still unclear how shifts in administering healthcare have impacted different patient populations [40-45]. The noted racial disparity identified in the current study is significant and could be the result of multiple factors, including uncaptured socioeconomic factors, an interaction present between race and insurance status, or an increased rate of COVID-19 infection in minority patients, prohibiting them from seeking out surgical care during the pandemic. Alternatively, the racial differences observed may reflect a difference in the medically necessary surgical diseases across races, although this would seem less plausible given the lack of data to support this. In this study, 96 $(19.6 \%)$ and 67 (18.3\%) Black patients underwent benign and oncologic procedures, respectively, in 2019 and 6 (9.2\%) and $13(8.8 \%)$ Black patients underwent benign and oncologic procedures, respectively, in 2020 , declines in absolute number from 2019 to 2020 were similar across benign and oncologic procedures. Further investigation is warranted to better understand the racial disparities in surgical care that appear to be magnified during the COVID-19 pandemic.

This study is one of the first to the authors' knowledge to examine perioperative outcomes of patients hospitalized during the COVID-19 pandemic for non-COVID-19 diagnoses. Other recent studies, performed among patients undergoing bariatric surgery, head/neck cancer resection, and colorectal surgical procedures, have noted that the overall safety profile of surgery during the COVID-19 pandemic is acceptable [46-48]. However, in one study of patients undergoing colorectal surgery, the postoperative mortality rate was noted to be higher during the pandemic [47]. The pandemic has caused significant redistribution of staff and resources at many institutions, including our own, resulting in changes in routine practices, which could have negatively impacted perioperative outcomes. Front-line healthcare providers have experienced higher fatigue and burnout during the pandemic, which could impact their ability to care for others during this time [49-51]. Even so, morbidity and readmission rates among those patients who underwent surgical procedures during the pandemic and in the year prior were not significantly different. Notably, the median length of stay for patients who underwent surgical procedures during the pandemic was shorter than that for patients who underwent surgical procedures prior to the pandemic, possible the result of clinicians' efforts to discharge patients from the hospital as soon as possible in order to mitigate infection risk and to prioritize hospital resources. The lower rate of discharge to skilled nursing facilities among the COVID-19 era patients likely reflects providers' efforts to keep patients out of healthcare facilities where exposure risk may be higher whenever possible. These findings are important given the ongoing need to maintain medically necessary surgical procedures nationwide and should be reassuring to patients undergoing medically necessary surgical procedures.

Our study is not without limitations. This study occurred at a single, large academic center with a large, diverse surgical census and our findings may not be generalizable 
to other facilities. Morbidity was measured as a composite of a large group of possible postoperative events that can occur for surgical patients, but each individual event may have a different weight depending on the particular procedure type. We attempted to mitigate this by ensuring that our match prioritized ICD-10 procedure code categories. With this approach, patients with ICD-10 procedure code categories that did not correlate to those found within our 2020 cohort in our matching algorithm were not included in the matching process, which may have skewed overall morbidity rates. Next, our cardinal matching approach matched each covariate based on prioritization; those covariates lower in the prioritization may have a weaker match as compared to those higher in prioritization. As such, this resulted in cohorts with residual differences in some patient and insurance differences which could impact findings of the adjusted perioperative outcomes. Additionally, our study is biased in that we only analyzed patients who were scheduled and underwent surgical procedures; we were not able to accurately capture those patients who may or may not have been seen in clinic but were unable to proceed to surgery. Inability to access outpatient pre-operative care or schedule medically necessary procedures could have influenced the findings of this study in an unknown manner. Although the current study is limited to capturing only those surgeries that were scheduled and performed during the study time period, a better understanding of which surgical procedures was postponed or rescheduled secondary to the pandemic and the rationale for this deference would be an important area of study. Patients seen in clinic may have had their surgeries postponed without clear documentation of the rationale or may have had their initial clinical appointments postponed, canceled, or never scheduled without documentation of the rationale. Given the effect of the pandemic on multiple non-surgical specialties, patients may have experienced a delay in diagnosis of new surgical conditions, which would be difficult to quantify.

The current study is limited by its inability to accurately capture the reasons to proceed with a particular surgical procedure (or not) during the early pandemic. The decision for surgery was ultimately left to the individual surgeon in careful discussion with the individual patient. These decisions were, in general, in accordance with the CMS, NCCN, and individual societies' guidelines and with oversight from the respective surgical department and division leadership with consideration of the safety of the patient and the healthcare providers' safety, need for timeliness of surgically addressing the underlying condition, and the availability of institutional resources. These were dynamic decisions as the pandemic unfolded, and unfortunately, cannot be captured in their detail through our institutional medical records. Additionally, emergency procedures were excluded from the study, making the results of the study potentially less generalizable. The authors elected to exclude these cases because, by their very nature, the decision to reschedule due to pandemic restrictions may not be a feasible option in emergency surgery, and consequently, any socioeconomic and racial disparities may therefore not be adequately highlighted through this procedure type, thus confounding the other findings of the study. Given the relatively small number and heterogeneous nature of the emergency cases that occurred during this time period (66 cases were designated as "hot" or "cold," identifying them as in the operating room within 2 and $6 \mathrm{~h}$ from case scheduling, including those cases booked both from the emergency room and inpatient), a meaningful subgroup analysis of these cases would not be feasible. Emergency surgery has been frequently associated with higher postoperative complications across a multitude of surgical procedures, further potentially confounding the results of this study $[52,53]$. How the COVID-19 pandemic affected patients' decisions to present to the emergency room with a potential surgical problem is an important area of future study, but one which could not be captured through the current medical records at our institution and under the current study design.

The COVID-19 pandemic has led to nationwide, largescale changes in access to healthcare. Our study notes that there appears to be no increased rate of morbidity, suggesting that non-emergent surgery for those with medically necessary needs can be performed safely during a pandemic. Even so, pandemic-related restrictions in surgical procedures appear to impact various races and insurance populations disproportionately. Racial- and insurancebased differences in those undergoing elective surgical care during the COVID-19 pandemic were observed, potentially reflecting underlying disparities which may warrant further study. Efforts to better understand this disparity and whether this may reflect an access problem (i.e., inability to attend in-clinic visits versus inability to gain access to telemedicine) or other impacts of the pandemic on minority and non-private insurance patients are urgently needed. Clinicians and policy makers should be conscious of the potential for insurance and patient disparities that may present during a national health crisis.

Funding No funding was provided for this study.

\section{Compliance with ethical standards}

Conflict of interest All authors have declare that they have no conflict of interest. 


\section{References}

1. Zhu N, Zhang D, Wang W, Li X, Yang B, Song J et al (2020) A novel coronavirus from patients with pneumonia in China, 2019. N Engl J Med 382:727-733

2. Yu P, Zhu J, Zhang Z, Han Y (2019) A familial cluster of infection associated with the 2019 novel coronavirus indicating possible person-to-person transmission during the incubation period. J Infect Dis 2020(221):1757-1761

3. Richardson S, Hirsch JS, Narasimhan M, Crawford JM, McGinn T, Davidson KW et al. (2020) Presenting characteristics, comorbidities, and outcomes among 5700 patients hospitalized with COVID-19 in the New York City Area. JAMA. https://doi. org/10.1001/jama.2020.6775.

4. Wang D, Hu B, Hu C, Zhu F, Liu X, Zhang J et al (2020) Clinical characteristics of 138 hospitalized patients with 2019 novel coronavirus-infected pneumonia in Wuhan. China JAMA 323(11):1061-1069

5. Guan W, Ni Z, Hu Y, Liang W, Ou C, He J et al (2020) Clinical characteristics of coronavirus disease 2019 in China. N Engl J Med 382:1708-1720

6. Holshue ML, DeBolt C, Lindquist S, Lofy KH, Wiesman J, Bruce $\mathrm{H}$ et al (2020) First case of 2019 novel coronavirus in the USA. N Engl J Med 382:929-936

7. Cucinotta D, Vanelli M (2020) WHO Declares COVID-19 a Pandemic. Acta Biomed 91(1):157-160

8. Coronavirus disease (COVID-2019) situation reports (xxxx) World Health Organization. https://www.who.int/emergencies/ diseases/novel-coronavirus-2019/situation-reports/. Accessed 19 May 2020

9. Cases, data, and surveillance (xxxx) Centers for Disease Control and Prevention. https://www.cdc.gov/coronavirus/2019-ncov/ cases-updates/cases-in-us.html. Accessed 19 May 2020

10. Diaz A, Sarac BA, Schoenbrunner AR, Janis JE, and TM Pawlik (2020) Elective surgery in the time of COVID-19. Am J Surg. https://doi.org/10.1016/j.amjsurg.2020.04.014.

11. Gao Y, Xi H, and L Chen (2020) Emergency surgery in suspected COVID-19 patients with acute abdomen: case series and perspectives. Ann Surg.https://doi.org/10.1097/SLA. 0000000000003961.

12. (2020) ACS guidelines for triage and management of elective cancer surgery cases during the acute and recovery phases of coronavirus disease 2019 (COVID-19) pandemic. American College of Surgeons. https://www.facs.org/-/media/files/covid19/ acs_triage_and_management_elective_cancer_surgery_during_ acute_and_recovery_phases.ashx. Accessed 19 May 2020

13. Bartlett DL, Howe JR, Chang G, Crago A, Hogg M, Karakousis $\mathrm{G}$ et al. (2020) Management of cancer surgery cases during the COVID-19 Pandemic: considerations. Ann Surg Oncol. https:// doi.org/10.1245/s10434-020-08461-2.

14. Stahel PF (2020) How to risk-stratify elective surgery during the COVID-19 pandemic? Patient Saf Surg 14(8):1-4

15. Mazzaferro V, Danelli P, Torzilli G, dit Busset MD, Virdis M, Sposito C (2020) A combined approach to priorities of Surgical Oncology during the COVID-19 epidemic. Ann Surg. https://doi. org/10.1097/SLA.0000000000004005.

16. Weise K, Baker M, Bogel-Burroughs N (2020) The coronavirus is forcing hospitals to cancel surgeries. The New York Times. https://www.nytimes.com/2020/03/14/us/coronavirus-covid-sur geries-canceled.html. Accessed 14 March 2020

17. Elster E, Potter BK, Chung K (2020) Response to COVID-19 by the surgical community. Surgery. https://doi.org/10.1016/j.surg. 2020.03.011.

18. Prachand VN, Milner R, Angelos P, Posner MC, Fung JJ, Agrawal N et al. (2020) Medically necessary, time-sensitive procedures: scoring system to ethically and efficiently manage resource scarcity and provider risk during the COVID-19 pandemic. J Am Coll Surg. https://doi.org/10.1016/j.jamcollsurg. 2020.04.011.

19. Tillmans G, Chenevas-Paule Q, Muller X, Breton A, Mohkam K, Duverf $C$ et al. (2020) Surgical outcomes after systematic preoperative SARS-CoV-2 screening. Surgery. https://doi.org/10. 1016/j.surg.2020.05.006.

20. Wadhera RK, Wadhera P, Gaba P, Figueroa JF, Maddox KEJ, Yeh RW et al. (2020) Variation in COVID-19 hospitalizations and deaths across New York City Boroughs. JAMA. https://doi. org/10.1001/jama.2020.7197.

21. Yancy CW (2020) COVID-19 and African Americans. JAMA 323(19):1891-1892

22. Price-Haywood EG, Burton J, Fort D, Seoane L (2020) Hospitalization and mortality among black patients and white patients with covid-19. N Engl J Med. https://doi.org/10.1056/ NEJMsa2011686.

23. Thebault R, Ba TA, Williams V (2020) The coronavirus is infecting and killing black Americans at an alarmingly high rate. The Washington Post https://www.washingtonpost.com/nation/ 2020/04/07/coronavirus-is-infecting-killing-black-americans-analarmingly-high-rate-post-analysis-shows/?arc404=true. Accessed 7 April 2020

24. Hooper MW, Nápoles AM, EJ Pérez-Stabl (2020) COVID-19 and racial/ethnic disparities. JAMA 323(24):2466-2467

25. Institute of Medicine (US) Committee on the Consequences of Uninsurance. Coverage Matters: Insurance and Health Care. Washington (DC): National Academies Press (US); 2001. 3, Who Goes Without Health Insurance? Who Is Most Likely to Be Uninsured? https://www.ncbi.nlm.nih.gov/books/NBK223657.

26. Zubizarreta JR, Paredes RD, Rosenbaum PR (2014) Matching for balance, pairing for heterogeneity in an observational study of the effectiveness of for-profit and not-for-profit high schools in Chile. Ann Appl Stat 8(1):204-231

27. Rosenbaum PR, Ross RN, Silber JH (2007) Mimimum distance matched sampling with fine balance in an observational study of treatmetnt for ovarian cancer. J Am Stat Assoc 102(477):75-83

28. Silber JH, Rosenbaum PR, Trudeau ME et al (2001) Multivariate matching and bias reduction in the surgical outcomes study. Med Care 39(10): 1048-1064

29. Rosenbaum PR, Rubin DB (1985) Constructing a control group using multivariate matched sampling methods. Am Stat 39(1):33-38

30. Cochran WG, Rubin DB (1973) Controlling bias in observational studies. Sankyha-Indian J Stat Ser A 35:417-446

31. Bishop YM, Fienberg SE, Holland PW, Light RJ, Mosteller F (1977) Book review: discrete multivariate analysis: theory and practice. Appl Psychol Meas 1:297-306

32. Rosenbaum PR (2007) Sensitivity analysis for m-estimates, tests, and confidence intervals in matched observational studies. Biometrics 63:456-464

33. Maritz J (1979) A note on exact robust confidence intervals for location. Biometrika 66:163-170

34. StataCorp LLC (2019) Stata: user's guide. Stata Press, College Station, Texas. https://www.stata.com/manuals/u.pdf. Accessed 20 May 2020

35. R Core Team (2019) R: A language and environment for statistical computing. R Foundation for Statistical Computing, Vienna, Austria. Published 2019. https://www.R-project.org. Accessed 1 July 2019

36. Lei S, Jiang F, Su W, Chen C, Chen J, Mei W et al (2020) Clinical characteristics and outcomes of patients undergoing surgery during the incubation period of COVID-19 infection. E Clin Med 21:100331 
37. Liang W, Guan W, Chen R, Wang W, Li J, Xu K et al (2020) Cancer patients in SARS-CoV-2 infection: a nationwide analysis in China. The Lancet 21:335-337

38. COVIDSurg Collaborative (2020) Mortality and pulmonary complications in patients undergoing surgery with perioperative SARS-CoV-2 infection: an international cohort study. The Lancet. https://doi.org/10.1016/S0140-6736(20)31182-X.

39. Eberly LA, Khatana SAM, Nathan AS, Snider C, Julien HM, Deleener ME et al (2020) Telemedicine outpatient cardiovascular care during the COVID-19 pandemic: bridging or opening the digital divide? Circulation 142:510-512

40. Chauhan V, Galwankar S, Arquilla B, Garg M, Di Somma S, ElMenyar, et al (2020) Novel coronavirus (COVID-19): leveraging telemedicine to optimize care while minimizing exposures and viral transmission. J Emerg Trauma Shock 13(1):20-24

41. Forbes RC, Solorzano CC, and BP Concepcion. Surgical telemedicine here to stay: More support from a randomized controlled trial on postoperative surgery visits. Am J Surg. 2020; Article in press. doi: https://doi.org/https://doi.org/10.1016/j.amj surg.2020.03.033.

42. Hakim AA, Kellish AS, Atabek U, Spitz FR, Hong YK (2020) Implications for the use of telehealth in surgical patients during the COVID-19 pandemic. Am J Surg. https://doi.org/10.1016/j. amjsurg.2020.04.026.

43. Smith WR, Atala AJ, Terlecki RP, Kelly EE, Matthews CA (2020) Implementation guide for rapid integration of an outpatient telemedicine program during the COVID-19 pandemic. J Am Coll Surg. https://doi.org/10.1016/j.jamcollsurg.2020.04. 030 .

44. Balzora S, Issaka RB, Anyane-Yeboa A, Gray DM II, May FP (2020) Impact of COVID-19 on colorectal cancer disparities and the way forward. Gastrointest Endosci. https://doi.org/10.1016/j. gie.2020.06.042.

45. Minkoff H (2020) You don't have to be infected to suffer: COVID-19 and racial disparities in severe maternal morbidity and mortality. Am J Perinatol 37:1052-1054
46. COVIDSurg Collaborative (2020) Head and neck cancer surgery during the COVID-19 pandemic: An international, multicenter, observational cohort study. Cancer

47. COVIDSurg Collaborative. Outcomes from elective colorectal cancer surgery during the SARS-CoV-2 pandemic. Colorectal Dis. 2020;00:1-18

48. Singhal R, Tahrani AA, Ludwig C, Mahawar K, GENEVA collaborators et al. (2021) Global 30-day outcomes after bariatric surgery during the COVID-19 pandemic (GENEVA): an international cohort study. The Lancet 9:7-9

49. Zhan Y, Zhao S, Yuan J, Liu H, Liu Y, Gui L et al (2020) Prevalence and influencing factors on fatigue of first-line nurses combating with COVID-19 in China: a descriptive cross-sectional study. Curr Med Sci 40(4):1-11

50. Lai J, Ma S, Wang Y, Cai Z, Hu J, Wei N et al (2020) Factors associated with mental health outcomes among health care workers exposed to coronavirus disease 2019. JAMA Netw Open 3(3):e203976

51. Lu W, Wang H, Lin Y, Li L (2020) Psychological status of medical workforce during the COVID-19 pandemic: a crosssectional study. Psych Res 288: 112936

52. To KB, Kamdar NS, Patil P, Collins SD, Seese E, Krapohl GL et al (2019) Acute care surgery model and outcomes in emergency general surgery. J Am Coll Surg 228:21-28

53. Sharoky CE, Bailey EA, Sellers MM, Kaufman EJ, Sinnamon AJ, Wirtalla CJ et al (2017) Outcomes of hospitalized patients undergoing emergency general surgery remote from admission. Surgery 162:612-619

Publisher's Note Springer Nature remains neutral with regard to jurisdictional claims in published maps and institutional affiliations. 